\title{
Remarques critiques sur l'historiographie des Choctaw
}

Critical remarks on Choctaw historiography

Comentarios críticos sobre la historiografía de los Chactas

\section{Benjamin Balloy}

\section{(2) OpenEdition}

Journals

Édition électronique

URL : https://journals.openedition.org/jsa/16953

DOI : 10.4000/jsa. 16953

ISSN : 1957-7842

\section{Éditeur}

Société des américanistes

\section{Édition imprimée}

Date de publication : 15 juin 2019

Pagination : $97-122$

ISBN : 978-2-902715-11-4

ISSN : 0037-9174

\section{Référence électronique}

Benjamin Balloy, "Remarques critiques sur l'historiographie des Choctaw », Journal de la Société des américanistes [En ligne], 105-1 | 2019, mis en ligne le 15 juin 2019, consulté le 04 septembre 2022. URL : http://journals.openedition.org/jsa/16953 ; DOI : https://doi.org/10.4000/jsa. 16953 


\title{
Remarques critiques sur l'historiographie des Choctaw
}

\author{
Benjamin BALLOY *
}

Les travaux de Patricia Galloway sur les Choctaw historiques du Sud-Est des États-Unis ont fortement mis l'accent sur la dynamique de construction de cette population, dans la lignée d'une historiographie influencée par l'ethnogenèse. L'appréhension des Choctaw au XVIII ${ }^{\mathrm{e}}$ siècle en sous-ensembles régionaux correspondant à des populations à l'origine ethniquement distinctes trouve ses effets les plus susceptibles de discussion dans la proposition, notamment formulée par Greg O'Brien, de lire le conflit interne aux Choctaw de 1748-1750, entre factions réputées l'une " pro-française », l'autre « pro-britannique », comme révélatrice d'une ligne de fracture ethnique entre segments trop récemment fusionnés. Cet article examine les indices textuels portant sur diverses formes de segmentation, et se propose de réhabiliter une approche plus sociologique de ces phénomènes. [Mots-clés : ethnogenèse, Choctaw, ethnohistoire, dualisme (organisation sociale), segmentation, XVIII ${ }^{\mathrm{e}}$ siècle.]

Critical remarks on Choctaw historiography. Patricia Galloway's historical work on the Choctaw of the Southeastern United States has mainly focused on their coalescent dynamic as a people. In such ethnogenetic perspective, it is commonly assumed that regional subdivisions of the Choctaw in the 18th century trace back to ancient ethnical boundaries. Furthering the "multiethnic confederacy" hypothesis, Greg O'Brien recently promoted the idea that the conflict of 1748-1750, between a so-called "pro-French" and a so-called "pro-British" faction among the Choctaw, could be analyzed as the persistent effect of such ethnogenesis. This article, through a close examination of early 18th century documentary evidence, questions these lines and calls for a better apreciation of the sociological aspects of such segmentation. [Key-words: ethnogenesis, Choctaw, ethnohistory, dualism (social organization), segmentation, 18th century.]

Comentarios críticos sobre la historiografía de los Chactas. Los trabajos históricos de Patricia Galloway sobre el pueblo Chacta del siglo XVIII, originario del Sureste de los Estados-Unidos, han principalmente destacado la dinámica de construcción de dicho pueblo, conformemente a una historiografía influenciada por la corriente de

* Doctorant IMM-LIAS, EHESS [benjaminballoy@ymail.com]. 
la etnogénesis. En dicha perspectiva, se admite que los agrupamientos regionales de los Chactas en el siglo XVIII reflejan antiguas fronteras étnicas. Basándose sobre esa hipótesis de los Chacta como “confederación multiétnica”, Greg O’Brien propuso que el conflicto de 1748-1750 entre dos facciones de los Chacta, los unos "profranceses" y los otros "pro-ingleses", se analizara bajo el prisma de una fractura étnica entre pueblos recientemente reunidos. El presente artículo, realizado a partir de un análisis de documentos que datan de principios del siglo XVIII, rechaza esa lectura etnicista y propone más bien de considerar el fenómeno de división bajo una dimensión sociológica. [Palabras-claves: etnogénesis, Chacta, etnohistoria, dualismo (organización social), segmentación, siglo XVIII.]

« Devant un texte ou un document, la postérité pourra avoir mille idées de questions que nous n'avons pas [...], mais l'érudition ne souffrira pas de cet allongement du questionnaire, car sa tâche se borne à faire savoir à partir d'où on ne sait pas »)

(Veyne 1971, p. 306).

En dehors des États-Unis, les populations historiques du Sud-Est de 1'Amérique du Nord n'ont jamais suscité qu'un intérêt modeste, pour ne pas dire nul, dans les débats d'anthropologie sociale ${ }^{1}$. C'est donc principalement à l'approche ethnohistorique que nous sommes redevables d'un progrès dans la compréhension des spécificités ethnologiques de ces sociétés. Parmi celles-ci, les Choctaw tiennent une place éminente, qui justifie assez l'importance que nous donnerons ici à la discussion minutieuse des développements de leur historiographie par deux chercheurs, Patricia Galloway et Greg O’Brien, dont les travaux font aujourd'hui autorité ${ }^{2}$.

1. En dehors des pages récentes consacrées par Emmanuel Désveaux aux Natchez de la basse vallée du Mississippi dans Quadratura americana (Désveaux 2001), pratiquement aucune recherche en langue française n'a porté sur cette aire culturelle, depuis les articles du début du $\mathrm{Xx}^{\mathrm{e}}$ siècle de Marc de Villiers, contributeur régulier du Journal de la Société des américanistes. Mes remerciements vont à Gilles Havard, Emmanuel Désveaux et AnneMarie Duranton-Crabol qui ont accepté de relire des versions préliminaires de ce travail et dont les suggestions bienveillantes ont été très appréciables. Les évaluateurs anonymes du Journal ont bien voulu par leurs conseils me donner l'occasion d'en ajuster la conclusion; qu'ils en soient remerciés.

2. On utilisera dans la suite du texte « Sud-Est » comme une « expression anachronique mais commode pour désigner l'ensemble régional composé des États actuels de la Caroline du Sud, de la Géorgie, de l'Alabama, du Mississippi et du Tennessee » (Havard 2016, p. 209). Le choix a été fait dans cet article de suivre la graphie anglo-saxonne "Choctaw " plutôt que celle française de «(T)Chacta », pour désigner ceux qui se nomment eux-mêmes aujourd'hui "Choctaw Nation ». De même, on utilisera de façon conventionnelle Choctaw et l'adjectif qui en découle, choctaw, sans en faire varier le genre ni le nombre. 
Une lettre de 1675 de l'évêque de Cuba, Gabriel Diaz Vara Calderón, contient la première attestation connue de "la Prov. ${ }^{a}$ de Chacta " (Wenhold 1936). Jusqu'à la fin du XVII ${ }^{\mathrm{e}}$ siècle, on ne fait encore que les deviner, silhouettes vagues apparaissant dans plusieurs documents conservés à Séville par l'intermédiaire desquels nous connaissons l'expédition avortée de l'espagnol Marcos Delgado, en 1686 (Boyd 1937, p. 12-28), en direction des berges du Mississippi. Il faudra attendre les sources françaises de la basse vallée du Mississippi, et notamment l'expédition de Henry de Tonti, en 1702, pour voir leur figure prendre quelque consistance et leur localisation commencer à se préciser ${ }^{3}$.

Patricia Galloway n'a pas manqué de souligner les difficultés documentaires, textuelles et archéologiques, que devrait affronter l'hypothèse de l'existence de cette entité, localisée entre la Tombigbee et les sources de la Pearl, avant le $\mathrm{XVI}^{\mathrm{e}}$ siècle (Galloway 1995, p. 15-20, 166-170). L'historiographie de cette population, bien documentée grâce aux travaux fouillés de Galloway et à ceux plus récents de Greg O'Brien, a mis l'accent, contre l'évidence ethnologique d'un bloc choctaw uniforme, sur la dynamique historique de leur formation et sur l'existence de divisions internes - orientation qui rattache ces auteurs à un courant important des études historiques de l'Amérique coloniale ayant fait de 1'ethnogenèse un objet privilégié (Boccara 2005; Giudicelli 2010; Kowalewski 2006). Tandis que Galloway cherche principalement à avérer cette segmentation en retraçant la « genèse des Choctaw » (Galloway 1995, 2006), O'Brien la considère comme acquise. C'est donc l'interprétation du phénomène lui-même qui joue un rôle critique pour la compréhension historique, en particulier celle du factionnalisme qui culmine dans le conflit entre partisans des Français et des Britanniques entre 1748 et 1750 (O’Brien 2002).

Le scénario proposé - un processus d'agrégation historique récent, que Galloway situe entre les $\mathrm{XVI}^{\mathrm{e}}$ et $\mathrm{XVII}^{\mathrm{e}}$ siècles, et qui ne se serait achevé qu'au début du XVIII ${ }^{\text {e }}$ siècle - repose sur plusieurs séries d'arguments, principalement sur la conjonction entre des indices textuels et des traces archéologiques (Galloway 1995, p. 353). Ce sont moins ces dernières que nous voudrions examiner attentivement ici - nous n'aurions pas la compétence pour le faire que les traces de diverses formes de segmentation qui transparaissent dans les textes, et le choix, délibéré semble-t-il, fait par ces deux chercheurs de formuler cette histoire en termes de grandes divisions régionales et ethniques (Galloway 1994, p. 514; O’Brien 2002).

L'exposé progressera en suivant deux lignes critiques ${ }^{4}$. La première critique part des difficultés qui s'opposent à la détermination des entités pertinentes qu'il

3. AN MAR/2JJ, 56, 20. Voir aussi AN F3, 24, fol. 38, Document daté du 20 juin 1702, signé d'Iberville.

4. On ne cherchera pas à confronter ces deux auteurs sur la totalité de leurs archives. On s'intéressera plus particulièrement à l'examen des sources françaises de la première moitié du XVIII ${ }^{\mathrm{e}}$ siècle, en particulier AN C13A. 
conviendrait de retenir pour rendre compte de la segmentation des Choctaw. L'opacité des sources, jusqu'à un certain point, complique l'évidence des divisions territoriales retenues par Galloway et O'Brien. Cette opacité ne retient pas réellement l'attention de ces auteurs; s'ils font cas de la tension perceptible autour des divisions, et - c'est même là un des principaux mérites de leurs approches $-\mathrm{s}$ 'ils font travailler ces tensions dans leurs historiographies, l'objet désigné n'est pas véritablement mis au centre de leurs réflexions. Division: le mot certes, mais la chose? De quoi parlent les documents? Quel(s) objet(s) devons-nous faire correspondre à ces termes qui disent « division »?

Nous sommes apparemment devant un problème classique de nomenclature (Bloch 1997; Ginzburg 2013), dont les enjeux critiques paraissent dépasser le seul intérêt ethnographique visant à améliorer notre compréhension des organisations sociales amérindiennes dans le Sud-Est. Étant donné le rôle central joué par cette question des divisions dans l'articulation de la pensée de Galloway et d'O'Brien, sur le plan de l'explication historique, les assises documentaires sur lesquelles reposent leurs arguments doivent être examinées attentivement. Nous avancerons en particulier que la lecture géographique qu'ils proposent explique imparfaitement les organisations socio-territoriales indigènes, dont elle recouvre et masque par un langage inadapté une réalité vraisemblablement plus complexe. Demeurent, en effet, plusieurs indices de la persistance de formations sociopolitiques proprement amérindiennes, antérieures au XVIII ${ }^{\mathrm{e}}$ siècle au moins, articulant plusieurs villages ou villes entre eux, dans un rapport de coordination, de subordination éventuellement. Ces formations politiques indigènes, quoiqu'incomplètement appréhendées dans les documents, nous donnent à voir un niveau de la réalité ethnographique peut-être plus pertinent pour approcher la praxis de l'espace social choctaw.

L'autre ligne critique porte sur l'examen du mouvement argumentatif qui amène $\mathrm{O}$ 'Brien à relier - au détriment des sources de la première moitié du $\mathrm{XVIII}^{\mathrm{e}}$ siècle - les hypothèses génétiques et ethniques de Galloway avec l'identification des factions choctaw qui s'affrontent lors du conflit de 1748-1750. Est-il licite de faire du factionnalisme l'effet inévitable de cette division ethnique et de cette dernière la faille nécessairement travaillée par les tensions de l'histoire coloniale? Au départ de cette position critique, il est vrai, nous reconnaissons une certaine défiance intellectuelle a priori devant la solution avancée par Galloway, encline à ethniciser les traces matérielles, et, par une tautologie risquée, à s'appuyer sur les traces matérielles pour identifier des différences ethniques ${ }^{5}$.

5. «Ein Volk, ein Topf [un peuple, un pot] », ironisaient déjà les critiques de Gustaf Kossinna. Le réflexe a une longue histoire, intellectuelle et surtout politique (Demoule 2014, p. 172-181). 


\section{Analyse des divisions territoriales}

\section{La géographie interprétée comme référence et comme code}

"Eighteenth- and nineteenth-century European and American observers 》, écrit O'Brien, "frequently noted that the Choctaws divided themselves into three principal districts, usually referred to as the Western (which included [1e village de] West Yazoo), Eastern, and Sixtowns Divisions »; une telle affirmation s'affranchit indéniablement des incohérences qui entachent les témoignages anciens mentionnant ces divisions - dans la première moitié du XVIII ${ }^{\mathrm{e}}$ siècle $^{6}$.

Nous proposons dans un premier temps de procéder à un examen plus systématique qu'il n'a été fait jusqu'à présent des témoignages documentaires relatifs aux divisions géographiques des Choctaw au XVIII ${ }^{\mathrm{e}}$ siècle. Sur les difficultés qu'a posées aux chercheurs la nature contradictoire des documents, il suffira de rappeler que Swanton, se rangeant aux conclusions précipitées de Halbert, missionnaire familier des Choctaw de l'Oklahoma au XIX siècle, a popularisé l'idée (Swanton 1931) d'une répartition régionale des Choctaw en au moins quatre unités - appelées « Southern or Sixtown division »; " Central »; «Western » et « Eastern »-, tandis que les documents français dans la première moitié du XVIII siècle ne mentionnent généralement que deux ou trois « cantons » ou « bandes ».

Une première difficulté tient à l'emploi - tant dans les sources anglaises que françaises -d'une terminologie fluctuante, dont il n'est pas toujours aisé de distinguer à quelle entité elle se rapporte ou l'échelle à laquelle se situe l'observateur. Au tout début de 1730, l'officier suisse Lusser, à la demande du gouverneur Périer, rejoint Régis du Roullet (militaire également) et le jésuite Michel Baudouin à Chicachaé, ville choctaw dans laquelle le missionaire réside (Villiers 1922b, p. 231). Lusser a laissé un journal de cette mission? ${ }^{7}$. On y apprend en particulier que, après avoir retrouvé les deux hommes, il entreprend une tournée dans les différents villages de «la nation des Chactas », vraisemblablement à la demande au gouverneur Périer, soupçonnant les réticences des différentes parties qui la composent de s'engager aux côtés des Français dans la guerre de répression que ceux-ci ont lancé contre les Natchez (Balvay 2008). Le « dénombrement» des villages qui termine le rapport de Lusser est structuré en deux colonnes: l'une pour la «Partie de l'est » et l'autre pour la « Partie de l'ouest ».

6. Certes, O'Brien s'intéresse surtout à la seconde moitié du XVIII ${ }^{e}$ siècle et, pour cette dernière, les documents renvoient de plus en plus nettement à une division tripartite. La position de Galloway est plus difficile à circonscrire et a d'ailleurs évolué avec le temps, comme on s'en rend compte à la lecture du recueil d'articles Practicing Ethnohistory (Galloway et Kidwell 2004, p. 499; Galloway 2006).

7. AN C13A, 12, fol. 100r-133v. 
Le père Baudouin est un observateur de premier ordre des Choctaw durant la première moitié du XVIII ${ }^{\mathrm{e}}$ siècle, puisqu'il s'établit à Chicachaé, village du sud du pays choctaw, de 1728 à 1751 ; cette longue présence parmi eux ne nous a toutefois laissé que peu de témoignages. Des lettres conservées, on a largement retenu les passages précieux pour la compréhension de leur organisation territoriale. Sur ce point, répondant à la curiosité de Salmon, ordonnateur nouvellement arrivé à la colonie, il résumait ainsi son sentiment en 1732:

La nation Chaktas est celle qui occupe le plus de terrain dans ce continent, on compte 42 villages de gens qui parlent entierrement la meme langue; Tous ces villages sont partagés en deux bandes que les Chaktas distinguent en apellant les uns villages de deça et les autres Villages de dela le marest plat, ce n'est cependant pas un marest mais un grand Ruisseau qui separe tous les villages Tchactas en deux parties presques egales, comme ce Ruisseau court Nord et Sud pour distinguer plus facilement les Villages nous nommons ceux qui sont du costé du Ruisseau Villages de la partie de l'Est, et ceux qui sont de l'autre costé Villages de la Partie d'oüest. Ces Villages sont fort etendües et eloignés les uns des autres ${ }^{8}$.

Certainement, il s'agit d'un passage remarquable dont le didactisme assuré laisse à première vue peu de doute sur la transparence de ce qu'il énonce. O'Brien y a lu un argument en faveur de son hypothèse ethnique (O'Brien 2002, p. 15). Évidemment, il convient de distinguer entre ce que nous sommes enclins à comprendre dans l'idée de division, et ce que les observateurs ont vu, qui présente en soi un écart significatif. Mais on doit en fait compliquer le modèle. Les documents ne sont pas seulement le moyen de réduire la distance qui nous sépare de l'objet, ils sont le lieu où se superposent et se nouent les perspectives d'intelligibilité de plusieurs objets (Ginzburg 1999, 2013, 2015).

Carlo Ginzburg, dans L'Inquisiteur comme anthropologue (Ginzburg 2010, p. 407-424) introduisait déjà cette idée que nous regardons par-dessus l'épaule d'un enquêteur doté de ses propres catégories conceptuelles, imposant aux voix silencieuses que nous essayons d'interroger à notre tour un premier niveau de distorsion, auquel s'ajoutent nécessairement les distorsions de nos propres catégories ou outils conceptuels. Et pourtant, défend Ginzburg, nous pouvons nous prémunir contre cette mise à distance qui - dans une optique positiviste aussi bien que « néo-sceptique »-semblera insurmontable, par la conscience critique de la « structure dialogique implicite » de ces textes. En mobilisant la distinction entre ce que Kenneth Pike (Ginzburg 2013; Sardan 1998) appelle niveaux « étique » - de l'observateur extérieur - et « émique » - des locuteurs eux-mêmes -, il est possible de penser, et donc partiellement neutraliser, les interactions inévitables entre les questions que nous posons à travers un texte et les questions auxquelles, dans l'intention de son auteur, le texte entend

8. AN C13A, 15, fol. 218v-219, publié partiellement dans (Villiers 1922b, p. 239). 
répondre, ouvrant une brèche par laquelle nous efforcer d'en atteindre les « ambitions référentielles ». Il nous semble qu'on ne peut saisir la réponse donnée à Baudouin et reformulée par lui, sans essayer de ressaisir, sinon la compréhension par les Choctaw de la question qui leur était posée, du moins les risques d'incompréhension inhérents à l'usage amérindien de codes topologiques pour formuler des configurations sociologiques.

En 1732, Baudouin était arrivé à la colonie depuis quatre années. Nous n'avons pas la preuve qu'il parlait la langue. Dans le passage cité, on perçoit une tension, fugace mais décisive, entre au moins deux discours, quoique ce soit la même voix, celle de Baudouin, qui les énonce. Le premier de ces discours rapporte un point de vue que l'on pourrait qualifier (Ginzburg 2013) de plus proprement émique («que les Chaktas distinguent»), qui rend compte de la manière dont se «partagent» du point de vue des Choctaw l'ensemble des villages « en deux bandes ». Les termes faussement évidents de la topographie pour formuler ce partage ( « de deça » et « de dela le marest plat ») ne doivent cependant pas nous égarer. Le recours au registre spatial pour signifier des oppositions sociologiques est un trait bien connu des systèmes amérindiens. Chez les Chickasaw et les Creek, populations voisines des Choctaw et appartenant au même ensemble linguistique muscoguéen, l'expression " the other side [of the fire] » sert dans un sens très concret à définir une opposition " Nous/Eux » (Nairne 1988, p. 43; Hewitt 1939, p. 128). Mais ce serait surtout passer à côté de l'importance accordée au marécageux, au boueux comme centre instituant dans les mythologies de ces sociétés. Une version du récit des origines des Choctaw, publiée en 1828 (Gatschet 1884, p. 106), commence ainsi:

When the earth was a level plain in the condition of a quagmire [un bourbier], a superior being, in appearance a red man, came down from above, and alighting near the centre of the Choctaw nation, threw up a large mound or hill, called Nanne Wayah, stooping or sloping hill.

De même, le récit de création des Creek fait une large place à la boue - " the stirring of living things in the mixture of the earth and the water 》-d'où les ancêtres émergent grâce à lotca, tortue; et aujourd'hui encore, là où les «stomp dances » ont lieu, la terre est aspergée d'eau et, du crépuscule au matin, invoquant l'aide de tortue, les danseurs, actualisent le voyage d'émergence originel (Chaudhuri et Chaudhuri 2001, p. 15-16).

Or, ce discours émique rapporté est immédiatement suivi par une reformulation, au niveau étique. Cette reformulation néglige la subtilité ethnographique signalée, laquelle est par conséquent recouverte, voire trahie, par la rationalisation que Baudouin - et les autres français avec lui sans doute puis à sa suite sûrement - opère des termes de la distinction (« pour distinguer plus facilement [...] nous nommons », « en deux parties presque égales »). Cette façon de prendre au premier degré la géographie (« ce n’est cependant pas un marest 
mais un grand Ruisseau ») - c'est-à-dire sans entrevoir qu'il puisse s'agir d'un code culturel à déchiffrer au lieu d'une référence limpide à corriger -, nous paraît rendre compte de l'invention et du succès durable chez les Français de la séparation des Choctaw en deux parties, d'est et d'ouest. Faute de ruisseau sur lequel fonder cette distinction - en dépit de l'affirmation de Baudouin -, les Français, nous allons le voir, ne parvinrent jamais à fixer avec sûreté la frontière entre ces deux parties, l'attribution des villages les plus centraux à l'une ou l'autre fluctuant entre les listes'.

Les critiques que nous formulons sur ce qui pose problème dans ces différents témoignages ne visent évidemment pas le principe d'une forme de division des Choctaw, mais plutôt notre capacité à caractériser le phénomène, à reconnaître l'objet ethnographique que les sources désignent. Nous avons suggéré plus haut, en commentant le passage de la lettre de Baudouin à Salmon, qu'une voix à la tonalité plus authentique, quoique nullement transparente, paraissait avoir filtré jusqu'à nous. C'est elle encore qu'on croit surprendre, mêlée d'une manière indécidable à celle de Baudouin, dans un passage de la correspondance de Salmon ${ }^{10}$ :

[Baudouin] est arrivé a la Nouvelle Orléans il y a environ un mois et j'ay conféré sur cela avec luy, il prétend que son calcul [recensement] est juste sur le fondement que tous les Tchactas sortent de deux races principalles, Sçavoir des Inoulakta qui est la plus nombreuse et la plus noble et des Eukatatlapé, qui est moins Considerable et moins distinguée. Il dit, que la première race est partagée en sept classes différentes et la seconde en cinq ce qui forme douze partis différents.

Dans son interprétation de ce passage, Swanton propose de traduire « eukatatlapé » par " yuka, captive, prisoner, slave, and tathlapi, five, the whole meaning apparently "the five captive or slave groups" " (Swanton 1932, p. 357). Il est cependant difficile d'admettre que Salmon, écrivant eu-, ait souhaité retranscrire [iu] - comme un anglophone pourrait l'avoir fait - au lieu de [Ø] ou de [u]. Par conséquent, il paraît plus prudent de reconstruire, sur le modèle de okla hannali (« les six villages »), un *okla tathlapi ${ }^{11}$.

Des deux « bandes », nous voici passés à deux « races », hiérarchisées et ellesmêmes segmentées. Certes, d'un témoignage à l'autre, pris à la même source

9. La carte la plus instructive à cet égard est sans aucun doute celle de 1743, conservée à la Library of Congress, intitulée «Carte particulière d'une partie de la Louisianne ou les fleuve et rivierres [i.e. rivières] onts etés relevé a l'estime \& les routtes [i.e. routes] par terre relevé \& mesurées aux pas, par les Srs. Broutin, de Vergés, ingénieurs \& Saucier dessinateur » (Demarigny 1743). Sur les cartes, l'ouvrage de référence reste Cumming 1998. Sur la démarche critique et les difficultés d'interprétation des récits de voyage et des cartes: Galloway 1995, 2006; Waselkov 1989.

10. AN C13A, 17, fol. 39v, Salmon, Lettre du 8 février 1733.

11. Voir également un okla untuklo « seven towns » (Swanton 1931, p. 81). La possibilité d'identifier chacun des termes à une moitié, selon un modèle d'organisation dualiste, et les " classes » à des clans est discutée dans la suite. 
et à seulement quelques mois d'écarts, l'apparence d'un principe dichotomique est conservée. Néanmoins, on a le sentiment d'avoir affaire, dans ce passage, à tout autre chose qu'à la reformulation sous un autre nom et mieux précisée, de l'objet visé dans la lettre précédente. Dans l'apparente transition d'une division territoriale à ce qui ressemble davantage à une division sociologique, qu'est-il légitime de conserver du point de vue précédent? Doit-on penser à un même objet saisi sous deux angles, qu'il suffirait d'articuler (comme le propose d'une certaine façon O'Brien) pour l'énoncer plus complètement ou, compliquant le jeu, faut-il postuler l'appréhension partielle et discontinue d'éléments territoriaux et sociaux qui participent d'organisations socio-politiques cohérentes mais fluctuantes et dont la teneur des articulations échappe largement aux observateurs?

\section{Discordance et contradiction des sources}

Afin de faire ressortir davantage les discordances et les contradictions entre les sources, nous procéderons avant tout à la mise en comparaison d'un type de document: les listes de villages, généralement établies dans un but de recensement, par des militaires français, dans la première moitié du XVIII ${ }^{\mathrm{e}}$ siècle (Swanton 1931).

C'est le cas de celles établies par Régis du Roullet autour des années 1730. Au retour de son troisième voyage en 1732 (Villiers 1922b; Galloway 2006), il dresse sa liste la plus précise - il avait déjà rendu compte des villages dans lesquels il avait été reçu et où il avait distribué des présents, lors de son premier voyage en 1729 -, et l'organise en trois «parties » ou « bandes »: 1) « partie de l'ouest que les sauvages nomment goula falaya, c'est-à-dire les amis de la grande bande », qu'il appelle plus loin « grande Bande ou Partie de l'Ouest »; 2) «Villages du centre de la Nation ou goula tchitou, les gros amis » et enfin, 3 ) « Partie de l'Est ou goula tennap qui veut dire les amis de l'autre côté » (Villiers 1922b). Indéniablement, les termes choctaw confèrent à sa liste un parfum d'authenticité auquel O'Brien pourrait avoir succombé. Roullet n'est cependant pas le seul à " nommer » ses divisions d'un terme indigène. Il y aurait, par conséquent, une certaine légèreté à identifier à l'Ouest, à l'Est et aux « six villages », respectivement « Okla Falaya », « Okla Tannap » et « Okla Hannali », termes sur l'identité desquels les sources, justement, restent foncièrement contradictoires - au moins pour les deux premiers. Certes, cette manière de partition en trois ensembles, qu'elle serve à transposer la perception de divisions réelles plus complexes ou qu'elle relève d'une invention des Français, continue d'apparaître avec un certain entêtement dans la documentation, mais là encore avec d'importantes variantes ${ }^{12}$. La systématicité du procédé, surtout à considérer superficiellement les listes, ne doit pas tromper et, dans un même

12. Le manuscrit français du XVIII ${ }^{\mathrm{e}}$ siècle, d'un auteur anonyme se trouve actuellement à la Newberry Library Chicago, Ayer ms. 530 « Anonymous Relation ». Galloway propose 
document, un observateur peut user avec plus ou moins de rigueur de ces divisions (Villiers 1922b, p. 240).

Dans les documents du milieu du XVIII ${ }^{\mathrm{e}}$ siècle où il est fait mention de ces distinctions géographiques, il n'est pas facile de décider si les catégories employées par les Français, et que ces derniers placent volontiers dans la bouche des Choctaw eux-mêmes ${ }^{13}$, relèvent d'un accomodement mutuel, une sorte de " middle ground " (White 2009). Le problème posé par l'articulation contradictoire entre formules binaires et trinaires, ainsi que celui des origines de cette division, était vraisemblablement ressenti par les contemporains eux-mêmes ${ }^{14}$.

Retenons plutôt que les divisions géographiques observées étaient majoritairement conçues par les observateurs du XVIII ${ }^{\mathrm{e}}$ siècle comme des entités - essentiellement territoriales et faiblement politiques -, englobant des unités de rang inférieur, les villages. C'est donc au niveau du village que paraissent converger les différentes sources, qui s'accordent pour l'identifier comme le lieu et l'objet privilégié des interactions diplomatiques et commerciales avec les Choctaw $^{15}$. Considérées dans le détail pourtant, les listes de villages se révèlent plus contradictoires encore.

Le cas de la division dite des « six villages » offre un aperçu illustratif des discordances entre les listes (voir Figure 1). Galloway et O’Brien ne précisent jamais vraiment quelles sont les villes qu'ils considèrent appartenir à l'une ou l'autre des divisions. Contre l'hypothèse du rattachement tardif de ce groupe aux Choctaw « originaux », on fera remarquer que le témoignage d'un membre de la première expédition d'Iberville incite à situer Chicachaé dans les environs, mêmes lointains, de Biloxi, dès 1698 (Pénicaut 1721, fº7).

Loin de résoudre les ambiguiés déjà mentionnées, la confrontation de ces listes à l'échelle des villes accentue le sentiment que la compréhension que les observateurs extérieurs pouvaient avoir des organisations sociales, territoriales et politiques des Choctaw, demeurait limitée. La lecture en termes géographiques traduit une appréhension imparfaite des organisations socio-territoriales indigènes, dont elle recouvre et masque par un langage inadapté une réalité certainement plus complexe. Ces discordances de détail pour dire une «division » des Choctaw, si évidentes à la confrontation des sources, ont un envers positif. Plusieurs arguments, que nous proposons d'examiner maintenant, incitent à conserver partiellement l'idée d'une forme de segmentation territoriale, en

une datation circa 1730; Swanton, plus tardif, vers 1750 (Swanton 1931; Galloway 2006, p. 204). Pour une autre liste de 1764 : Rowland 1911, p. 26-28.

13. AN C13A, 30, Journal de Beauchamp de 1746, fol. 228, 229v.

14. Dans un rapport rédigé en 1758, « sur les Peuplades des vallées du Mississipi et du Missouri » (Villiers 1907, p. 76-77), le chevalier de Kerlérec, gouverneur de la Louisiane, formule par exemple cette articulation en termes historiques.

15. Voir également (Piker 2004, p. 3) qui souligne ce point pour les Creek (Muskogee). 
Remarques critiques sur l'historiographie des Choctaw

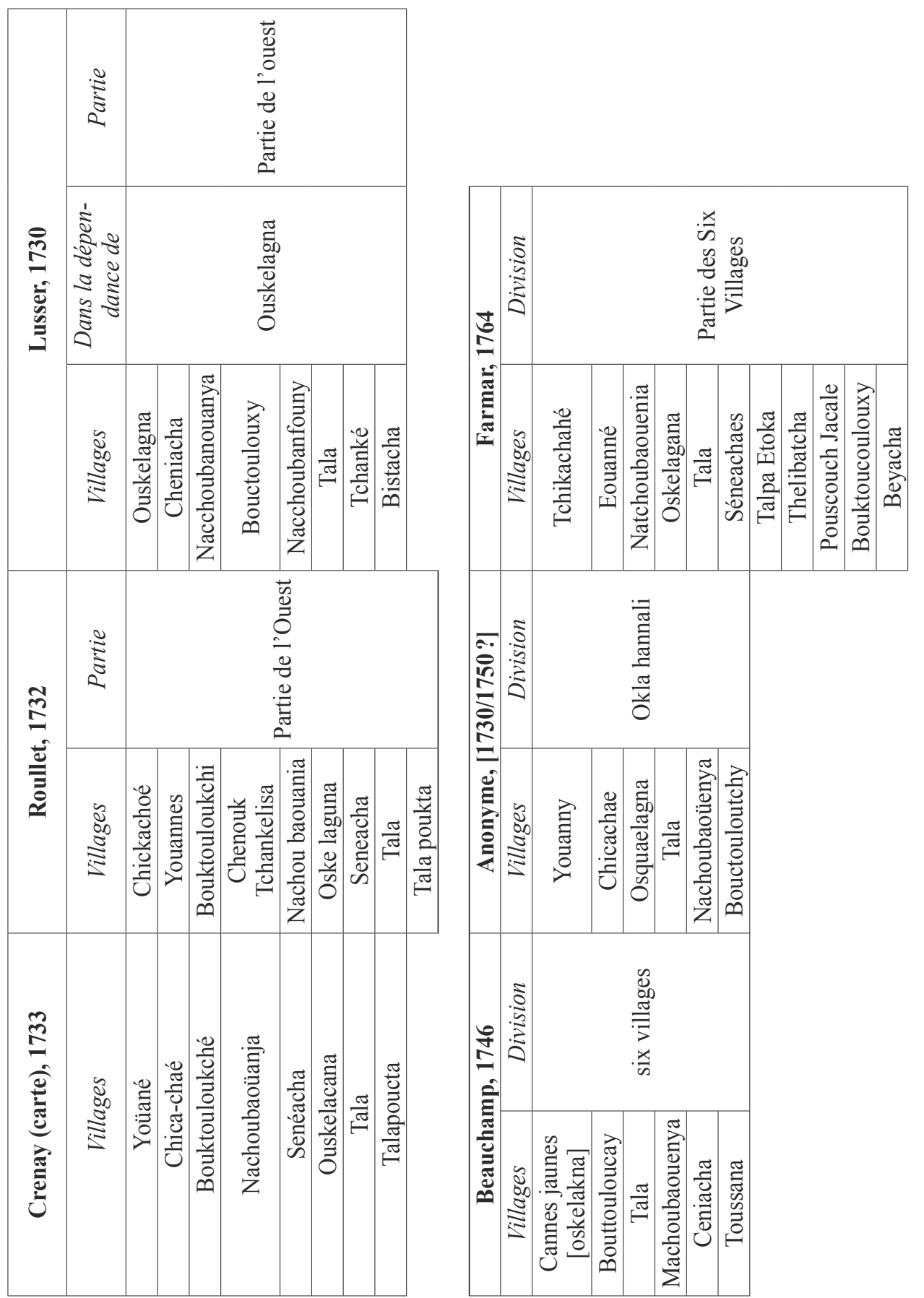

Fig. 1 - Comparaison des listes de villages du XVIII ${ }^{\mathrm{e}}$ siècle qui mentionnent la division des « six villages ». 
cherchant à faire émerger un niveau intermédiaire entre le village et les trop vastes unités géographiques des observateurs européens du XVIII ${ }^{\mathrm{e}}$ siècle.

\section{Traces d'une continuité avec les formations antérieures au XVIII siècle}

À propos de la communauté de Yazou de l'Ouest en 1764, O'Brien a introduit l'expression de « communauté ou groupement de villages » (village clusters; O’Brien 2002, p. 12-13). Galloway a pu parler à l'occasion de " groupings » ou « groups » de villages; mais ce qu'O'Brien identifie ici, c'est un niveau intermédiaire entre les pseudo-divisions géographiques et les villages, là où Galloway réconciliait, sans doute de façon trop peu critique par rapport aux sources, lesdites pseudo-divisions avec ces entités (Galloway 1995, p. 2). Plutôt que de diluer la spécificité de cette forme d'organisation dans des unités territoriales aux contours fluctuants et dont on peine à déterminer s'ils cernent véritablement un aspect de la praxis amérindienne, un tel niveau d'analyse ménagerait une voie intermédiaire que l'ethnohistoriographie pourrait s'employer à rendre effective (Hann 1988, p. 102; Kowalewski 2006, p. 105).

Il nous semble que la liste de Lusser de 1730 en offre l'attestation la plus ancienne et la plus complète - caractéristique sur laquelle, étonnamment, les commentateurs ne se sont pas attardés. Si sa liste des villages Choctaw est présentée selon le principe de division géographique déjà évoqué, elle s'enrichit d'un second niveau de segmentation, clairement perçu comme relatif à un niveau intermédiaire d'organisation territoriale des Choctaw qu'il a visités. Ces sous-divisions ne sont pas nommées, mais chaque fois regroupées par une accolade que précède le nom du village principal «avec ses dépendances ». Ainsi, sur les 47 entrées que comporte la liste de Lusser en 1730, et si l'on excepte les 3 derniers villages qui sont ceux des « Chactas Etably sur la Riviere de laMobile », les 44 villages qu'il recense se laissent distribuer en 15 sousensembles: 11 pour la partie de l'est et 4 pour celle de l'ouest (voir Figure 2). Ce même terme de « dépendance » revient également à plusieurs reprises sous les plumes de Roullet ou de Beauchamp.

Fig. 2 - « Nom des Villages Chactas et denombrement que chacun de ses Villages peut contenir d'hommes portant les armes... ", attribué à Lusser, d'après AN C13A, 12, fol.133. 


\begin{tabular}{|c|c|c|c|}
\hline Nom (des villages) & $\begin{array}{l}\text { Hommes portant } \\
\text { les armes }\end{array}$ & Ensemble & Partie \\
\hline Ouanis & 50 & Ouanis & \multirow{28}{*}{ Partie de l'est } \\
\hline Chicachaé & 160 & Chicachaé & \\
\hline Conchas & 100 & \multirow{6}{*}{ Conchas } & \\
\hline Abissa & 30 & & \\
\hline Boucfalaya & 15 & & \\
\hline Panty & 70 & & \\
\hline Ouantonoullou & 30 & & \\
\hline Boucchito & 20 & & \\
\hline $\begin{array}{l}\text { Couenchito, Village } \\
\text { du grand chef }\end{array}$ & 80 & \multirow{5}{*}{ Couenchito } & \\
\hline $\begin{array}{c}\text { Louckata } \\
\text { [Loucfeata] }\end{array}$ & 60 & & \\
\hline Choukelissa & 30 & & \\
\hline Bitoupgoula & 30 & & \\
\hline Itéchipouta & 60 & & \\
\hline Bouctoucoulou & 130 & \multirow{3}{*}{ Bouctoucoulou } & \\
\hline $\begin{array}{c}\text { Alibamon } \\
\text { Chougalougol }\end{array}$ & 40 & & \\
\hline Yajoux & 70 & & \\
\hline Scanapa & 180 & \multirow{7}{*}{ Scanapa } & \\
\hline Atlepelé & 50 & & \\
\hline Bitoupgoula & 100 & & \\
\hline Apeca & 100 & & \\
\hline Iteotchako & 100 & & \\
\hline Kouctacanlé & 10 & & \\
\hline Mongoulacha & 20 & & \\
\hline Okeloussa & 100 & Okeloussa & \\
\hline Yanabé & 60 & Yanabé & \\
\hline Oulictacha & 50 & Oulictacha & \\
\hline Apeca & 50 & Apeca & \\
\hline Pousecouchelacalé & 80 & Pousecouchelacalé & \\
\hline Ouskelagna & \multirow{2}{*}{200} & \multirow{8}{*}{ Ouskelagna } & \multirow{8}{*}{ Partie de l'ouest } \\
\hline Cheniacha & & & \\
\hline Nacchoubanouanya & 50 & & \\
\hline Bouctoulouxy & 30 & & \\
\hline Nacchoubanfouny & 20 & & \\
\hline Tala & 60 & & \\
\hline Tchanké & 20 & & \\
\hline Bistacha & 15 & & \\
\hline
\end{tabular}




\begin{tabular}{|c|c|c|c|}
\hline Nom (des villages) & $\begin{array}{l}\text { Hommes portant } \\
\text { les armes }\end{array}$ & Ensemble & Partie \\
\hline Mongoulacha & 100 & \multirow{2}{*}{ Mongoulacha } & \multirow{8}{*}{ Partie de l'ouest } \\
\hline Okeoullou & 20 & & \\
\hline Boucfouca & 130 & \multirow{2}{*}{ Boucfouka } & \\
\hline Fanianke & 20 & & \\
\hline Kastacha & 120 & \multirow{3}{*}{ Kastacha } & \\
\hline Yasoux & 60 & & \\
\hline Atchouchouga & 20 & & \\
\hline Kasetalaya & 130 & Kasetalaya & \\
\hline Tomé & 60 & \multirow{3}{*}{\multicolumn{2}{|c|}{ Choctaw sur la Mobile }} \\
\hline Nanyaba & 50 & & \\
\hline \multirow[t]{2}{*}{ Mobiliens } & 30 & & \\
\hline & 3010 & \multicolumn{2}{|c|}{$16+1$} \\
\hline
\end{tabular}

Dans plusieurs cas, nous savons que le nom d'une ville servait tantôt à désigner un village stricto sensu, tantôt la "province » réputée lui être subordonnée (Hudson et Tesser 1994, p. 228, 246, 385; Kowalewski 2006, p. 107). Nous pouvons par exemple appréhender la réalité de ces ensembles politiques et territoriaux à travers les circuits de redistributions de cadeaux entre entités en direction desquelles les « chefs », en les représentant, font signe (Galloway 1994, p. 517, 521). S'il ne faut donc pas prendre trop au sérieux le découpage politique tel que le choix de distribuer des présents à un nombre restreint de chefs semble l'impliquer, il convient néanmoins de reconnaître, malgré un certain arbitraire, la présence de divisions réelles des Choctaw, en unités relativement autonomes et plus nombreuses que deux ou trois. Contre le fonctionnement politico-territorial centralisé et monarchique que les Français ont cherché à imposer aux Choctaw, on voit continuer à fonctionner, en 1732, ce réseau de " petites républiques ", mieux définies comme autant de $"$ principaux Villages $»^{16}$. O'Brien, qui cite le mot de Baudouin, ne rapproche pas cette dernière expression de son idée de « village clusters » mais en revient aux « trois divisions » (O'Brien 2002, p. 20). Il est ainsi remarquable que sur les 17 villages visités par Roullet lors de son premier voyage en 1729 , dans lesquels il a rencontré des chefs auxquels il a distribué des présents, il soit possible de retrouver 13 des 16 villages auxquels Lusser prête une forme de souveraineté.

Ce lien étroit entre entités demeurées distinctes - qui est perçu comme une forme de dépendance -, Beauchamp l'illustre en soulignant en 1746 la place importante conférée à Chicachaé dans l'ensemble des « six villages », discuté 
plus haut ${ }^{17}$. Déjà Noyan en $1739^{18}$, écrivait à propos des chefs de Conchas et de Chicachaé: « ils tiennent tous les deux un rang fort distingué dans la nation, [...] ayant l'un neuf et l'autre sept villages sous sa domination ».

Suivant la voie suggérée par O'Brien, on peut penser que les divisions renseignées par Lusser en 1730 doivent être interprétées comme l'indice de la persistance de formations sociopolitiques proprement amérindiennes, antérieures au XVIII ${ }^{\mathrm{e}}$ siècle au moins, et dont les fondements pourraient bien être rituels, articulant plusieurs villages ou villes entre eux, dans un rapport de coordination, de subordination possiblement - du moins selon une forme de relation que la notion forgée par Louis Dumont d'englobement hiérarchique permettrait certainement de mieux définir (Dumont 1978). Il y a là une piste de travail importante. Soulignons toutefois que cela implique de retenir les divisions renseignées par Lusser non pas comme l'indication des limites effectives de ces formations politiques indigènes, mais plutôt comme l'appréhension d'un niveau de la réalité ethnographique pertinent pour approcher la praxis de l'espace social choctaw.

Bandes, parties, cantons, races, nations, villages: il est donc clair qu'au $\mathrm{XVIII}^{\mathrm{e}}$ siècle, contrairement à ce qu'affirme O'Brien, les témoignages se présentent d'une manière trop contradictoire entre eux pour pouvoir 1) établir que l'organisation territoriale des Choctaw, dès le début du XVIII siècle, consistait en trois divisions plutôt qu'en deux - ou davantage; mais également, 2) admettre que tous les témoignages se référant à une division, quand bien même ils l'expriment en termes géographiques, par une transparence bien étonnante désigneraient nécessairement un objet aux contours identiques.

\section{Revenir à la sociologie contre la réduction du factionnalisme à des bases ethniques}

\section{La question des partis " pro-français » ou " pro-britannique »}

Cette tension entre des modèles tripartite et binaire, qui est le reflet direct de l'indécision des sources et de leurs apparentes contradictions, n'est aucunement résolue et agit même comme le point inarticulé de l'argumentation d'O'Brien. On y trouve en effet le passage, abrupt, sans commentaires, entre le modèle à division géographique tripartite - emprunté à Galloway dans ses présupposés ethnicistes et fusionnistes ${ }^{19}$ - et un nouveau modèle cette fois à deux populations

17. AN C13A, 30, fol. 227v.

18. AN C13A, 24, fol. 227. Baudouin, en 1742, écrit de même: «Les Chicachaés et les six villages qui en dependent...» (AN C13A, 27, fol. 131, 1v).

19. S'il emprunte à Galloway l'idée de fusion de populations originellement distinctes, auxquelles correspondraient les divisions historiques des Choctaw, il ne semble pas reprendre 
(O’Brien 2002, p. 21). Un tel mouvement paraît tenir à la volonté de trouver une base ethnologique à un phénomène historique, de faire coïncider deux ordres de phénomènes, perceptibles dans les sources, et de faire jouer à celui-là le rôle historiographique d'explication de celui-ci.

D'un côté, on trouve en effet, à partir notamment des sources plus tardives du $\mathrm{XVIII}^{\mathrm{e}}$ siècle, une forme d'opposition entre deux « groupes », sans bases territoriales apparentes, les uns désignés comme «Imonclacha », les autres comme « Inhulahta ». Ces termes continuent d'apparaître jusqu' au XIX ${ }^{\mathrm{e}}$ siècle, et c'est sur la base des informations transmises par des missionnaires des Choctaw de 1'Oklahoma - Cyrus Byington, Henry S. Halbert, Horatio B. Cushman... - que Swanton a proposé de les interpréter comme les désignations choctaw de leur « moitiés », en référence à un principe d'organisation dualiste. De l'autre, il y a l'existence, également documentée, d'un clivage au sein de l'ensemble Choctaw, que les Français furent prompts à radicaliser sous la forme d'un factionnalisme, entre les villes « pro-françaises » et celles réputées entretenir des velléités d'alliance ou au moins une faiblesse coupable envers les traiteurs britanniques de Charleston et leurs alliés chickasaw. Cette tension connait plusieurs épisodes au XVIII ${ }^{\mathrm{e}}$ siècle, mais elle paraît culminer dans ce qu'il est convenu d'appeler la « guerre civile » choctaw, qui voit en apparence s'affronter les deux camps, entre 1748 et 1750 . Celle-ci fait immédiatement suite à la guerre intercoloniale, dite « du roi George », entre Français et Britanniques, mais elle doit être inscrite dans une perspective plus large. Comme l'écrit Richard White, les Français «mettaient leurs problèmes sur le compte d'un complot britannique, et les Anglais encourageaient en effet très clairement la rébellion dans la région de l'Ohio et au-delà. Mais les causes de ces troubles étaient bien plus profondes. Les Français eux-mêmes avaient semé les graines de la révolte généralisée lorsque la Couronne chercha à faire des économies durant les quelques années qui avaient précédé la guerre [1744]» diminuant " de manière significative la quantité des présents offerts » et des marchandises disponibles dans les magasins (White 2009, p. 282-288).

La proposition d'O'Brien consiste à rapprocher les deux ordres de phénomènes, et à les articuler dans une trame historiographique synthétique. D'abord, afin d'inscrire ce mouvement dans la conjecture historique de plus longue durée de Galloway, il propose d'ethniciser les deux groupes: "Imoklashas and Inhulahtas reflected distinct ethnic groups ", lesquels " joined forces sometimes between 1540 and $1700 »$; ensuite, par une opération qui entretient avec le mythe des rapports étroits (Swanton 1929, p. 43; 1931, p. 78), il identifie chacun des groupes avec l'une des divisions territoriales: " with few exceptions, the Imoklasha ethnic group dominated the Western Division and the Inhulahta

à son compte les modèles plus complexes qu'elle a élaborés (Galloway 1989; 1994, p. 514515; 1995, p. 354; Galloway et Kidwell 2004). 
group controlled the Eastern Division " ; enfin, il peut identifier les factions ${ }^{20}$ qui se sont affrontées lors du conflit de 1748-1750 comme deux populations ethniquement distinctes et expliquer le conflit par leur origine ethnique distincte (O’Brien 2002, p. 15, 16, 20).

La pièce décisive du raisonnement, celle qui assure la liaison dans son dispositif historiographique entre l'hypothèse génétique des Choctaw comme fusion d'éléments ethniques disparates territorialisés et le factionnalisme identifiable à des divisions géographiques congruentes, est la liste de villages copiée par le cartographe Claude Delisle et attribuée à Iberville, datée de 1702 (AN MAR/2JJ, 56, 24 ; Villiers 1922a; O'Brien 2002, p. 16). L'interprétation d'O'Brien repose cependant sur une lecture fautive du terme " Ougahouslasla Chaquela », entête de la seule première moitié de la liste. Pour lui, "Imoklasha appears as "Ougahouslasla" ". La graphie française déforme sans nul doute les phonèmes originaux ainsi transcrits. Publiant cette liste, Villiers notait surtout que « la façon dont Claude Delisle traçait parfois ses $t$ rend souvent leur distinction difficile avec les $1 »$. La consultation de l'original permet en effet de rétablir la transcription suivante: "Ougahouslasta Chaqueta », qui se laisse mieux expliquer en okla hulahta chaqueta [groupe + hulahta + Choctaw] ${ }^{21}$; c'est-àdire exactement l'inverse de ce qu'O'Brien croit y lire.

En soulignant l'erreur d'interprétation de ce document, nous ne cherchons évidemment pas à suggérer qu'il suffirait d'inverser les termes pour remettre sur pied la série des identités conjoncturelles, mais seulement que ce document nous laisse dans l'indétermination quant à l'assertion (O'Brien 2002, p. 17) selon laquelle l'Ouest s'identifierait préférentiellement aux imonklasha et, partant, l'Est aux inhulahta.

C'est d'ailleurs l'insistance à apparier les termes eux-mêmes qui peut être contestée. Si inhulahta, on le sait, est attesté dès 1702, le terme d'imonklasha ne jouit pas de la même ancienneté et on ne voit pas bien à quelles sources O’Brien se réfère lorsqu'il écrit: " the Imoklasha and Inhulahta designations persistently appear in the documentary records as ethnic groupings that predate the Choctaws as a people » (O'Brien 2002, p. 15). De plus, en présentant l'opposition imonklasha/inhulahta comme une donnée ethnographique objective, O'Brien passe sous silence la sélection qu'il opère au détriment d'autres termes d'opposition possibles. Lorsque les missionnaires s'intéresseront à l'organisation sociale des Choctaw au XIX ${ }^{\mathrm{e}}$ siècle, imonklasha ne sera ni le seul terme,

20. Notons d'ailleurs que les opinions de Kerlérec (Villiers 1907, p. 76-77), et avant lui Baudouin déjà en 1732 (AN C13A, 15, fol. 231v), entrent en contradiction avec ce qu'O'Brien semble tenir pour acquis.

21. Sur la reconstitution de l'expression en okla $+\ldots$, voir toutes les expressions construites de cette façon: okla falaya, okla hannali, okla cito, okla tannap, etc. 
ni même le plus fréquemment apparié à inhulahta ${ }^{22}$. Et, l'opposition la plus anciennement attestée dans les sources reste, comme on l'a vu, celle du passage d'une lettre de Salmon du 8 février 1733, dans lequel ce n'est pas imonklasha mais l'expression d'interprétation délicate eukatatlapé qui est donnée.

Ajoutons qu'O'Brien n'affronte jamais sérieusement le fait que, dès le début du XVIII ${ }^{\mathrm{e}}$ siècle, « mongoulacha » surgit dans les documents français, comme nom d'une population localisée à proximité du Mississippi (Swanton 1946, p. 152, 176) ; dès la fin du XVII ${ }^{\mathrm{e}}$ siècle, dans les documents espagnols (Wenhold 1936; Boyd 1937) puis jusqu'au XIX ${ }^{\mathrm{e}}$ siècle, c'est également le nom d'une ville des Indiens alibamon (Imocolâsa, Miculasa...) ; et chez Nairne, apparaît de façon problématique dans l'apparente énumération de ce qu'on identifie comme des clans des Chickasaw, le terme « Muclesa » (Nairne 1988, p. 39). On pourrait en dire autant du terme holahta qui apparaît dès le $\mathrm{XVI}^{\mathrm{e}}$ siècle dans les documents espagnols comme un titre indigène (Hudson 2005), demeuré jusqu'ici relativement opaque, et qui réclamerait donc, pour le moins, une analyse ethnologique dépassant le seul cas des Choctaw.

On soulignera également que les documents ne disent jamais qu'une ville ou une division est inhulahta, et a fortiori, imonklasha; ce sont toujours des personnes qu'on identifie ainsi, qu'il s'agisse d'un individu ou d'un collectif23. Ajoutons que cette identité est définie en termes de « race » ou de « caste », ce qui n'est pas sans évoquer un groupe de parents ${ }^{24}$ et paraît en fait qualifier sous la plume des observateurs ce qui relie entre elles des personnes, sur un mode singulier et exclusif $^{25}$. Rien n'autorise dès lors à généraliser cette identité revendiquée par des individus à l'ensemble de la population d'une ville. De la même façon, il est difficile de préciser si le lien qu'on observe apparemment entre une faction et l'une de ces « races » possède la systématicité que lui prête O'Brien.

Enfin, dans les documents plusieurs observations rapportées contrarient le jeu supposément uniforme d'un factionnalisme à base ethnique tel qu'O'Brien l'envisage. Alibamon mingo, le chef de Conchas, est l'un des chefs choctaw les mieux singularisés dans les sources françaises (Galloway 2006). Il affirme en 1765 être de la "great race » des inhulahta, ethnie réputée associée aux villes de l'Est et indéfectiblement liée aux Français. Pourtant, ses revirements

22. Lewis Morgan (via Byington), Wright ou Halbert mentionnent plus volontiers le terme de kashapa: Swanton 1931, p. 78-79; Gatschet 1884, p. 104-105.

23. Rowland 1911, p. 236, 239; AN C13A, 17, fol. 39v, Lettre du 8 février 1733 de Salmon au Ministre.

24. Conformément aux usages français des XVI ${ }^{\mathrm{e}}$ et XVII ${ }^{\mathrm{e}}$ siècles: Havard 2009, p. 37.

25. Noyan notamment utilise la deuxième expression: AN C13A, 24, fol. 224. Dans une lettre de 1742 de Baudouin, on trouve: « [...] il fut a 4 heures du matin Chez Paymîngo son frere de race et son gendre... » (AN C13A, 27, fol. 131, 3v). Voir également une lettre de Beauchamps: AN F3, 24, fol. 448v. 
successifs entre 1736 et 1738 puis à nouveau en $1745^{26}$, incitent à nuancer considérablement les protestations d'attachement aux Français qu'il donne à Beauchamp en 1746 et à envisager avec prudence l'affirmation d'un lien étroit entre le fait d'être inhulahta et indéfectiblement attaché au parti français.

Quant au parti du chef séditieux Soulier Rouge qui en 1746 continue de traiter avec les Britanniques, l'examen attentif du journal de Beauchamp révèle plutôt que les positions étaient loin d'être aussi nettement décidées et, lorsqu'elles le sont, on ne saurait soutenir que les clivages recoupent nécessairement les divisions géographiques ou un quelconque principe ethnique ${ }^{27}$.

En 1746, beaucoup de chefs préférent encore afficher une position de neutralité à l'égard du « mutin » Soulier Rouge, connu également par le titre d'Imataha Tchito. C'est d'ailleurs dans ces termes, - soutenir ou s'opposer à Imataha Tchito et à son projet d'ouvrir les Choctaw à la traite anglaise, et non soutenir l'une ou l'autre puissance européenne - que sont généralement formulées les positions des chefs que Beauchamp rencontre; et c'est bien parce qu'il s'agit d'une condamnation des actions d'un parent éventuel que la plupart préfèrent ne pas s'engager dans cette voie. C'est le cas par exemple de l'étrange et véhémente fin de non-recevoir que Mongoulacha mingo, chef du village de Chicachaé, oppose aux exigences des Français. Le village est pourtant présenté comme pro-français, et il le sera en effet ensuite dans le conflit de 1748. Son attitude n'est compréhensible que si on la rapporte aux liens de parenté qui l'unissent avec le «mutin ». Imataha Tchito et lui, quoique Vaudreuil le note seulement en passant, sont des beaux-frères ${ }^{28}$.

« Il falloit laisser agir la nation », ne cessent de répéter les Choctaw qui s'adressent publiquement à Beauchamp en 1746, tant il est vrai - par le jeu complexe des réseaux d'alliance devons-nous comprendre - que « le mutin Imataha Tchitou avoit Beaucoup de partisans dans leur nation ${ }^{29} »$. Ces réseaux nous paraissent dessiner des postures plus lâches et circonstanciées que la tranchante alternative à laquelle les labels « pro-français » et « pro-anglais » tendent à les réduire. En tant que beau-frère, Mongoulacha mingo n'est pas un « partisan » d'Imataha tchito. Sa réaction traduit plus certainement l'inconfort de sa position, tiraillé par une fidélité indécidable envers deux sortes d'alliés incompatibles ${ }^{30}$.

26. AN C13A, 21, fol. 122, Lettre du 10 février 1736 de Bienville au Ministre; AN C13A, 23, fol. 95; AN C13A, 24, fol. 35; AN C13A, 29, fol. 189, Lettre du 6 octobre 1745 de Louboey au Ministre.

27. AN C13A, 30, fol. 232v; Vaudreuil évoque en 1749 les « Chactas de l'est divisés entre eux » (AN C13A, 33, fol. 12). Voir également ce qu'Alibamon mingo affirmait en 1738 dans un discours rapporté par Noyan: AN C13A, 24, Lettre du 4 janvier 1739, fol. 232v.

28. AN C13A, 32, fol. 122, Lettre du 5 novembre 1748 de Vaudreuil au Ministre.

29. AN C13A, 30, fol. 222v.

30. AN C13A, 30, fol. 225v. De fait, ce sera également le destin de Mongoulacha mingo d'être « tué par cinq guerriers de son village qui l'ont puni de la proposition qu'il avoit 


\section{Réintroduire le dualisme}

L'assimilation des termes choctaw, imonklasha et inhulahta, à des désignations ethniques suppose par ailleurs le rejet explicite du modèle sociologique dualiste, majoritairement adopté pour décrire, dans ces sociétés, les systèmes de moitiés (O'Brien 2002, p. 15). Les arguments pour motiver ce rejet se fondent sur une compréhension hasardeuse de la teneur et des formes du dualisme sociologique dans les sociétés du Sud-Est, sinon sur une théorie du dualisme inadéquate $^{31}$ - grief que lui adresse également Galloway avec des arguments pertinents (Galloway 2006, p. 358-59).

Assurément, notre compréhension de l'organisation sociale des Choctaw, à l'instar de celle des Chickasaw et - dans une moindre mesure - des Creek, demeure extrêmement lacunaire. La synthèse à laquelle Swanton s'est essayé laisse en friche un grand nombre de questions (Swanton 1931, p. 57-80), comme l'ont prudemment noté les auteurs de l'article du Handbook of North American Indians (HBNAI) :

The three social units-moieties, iksas, and local groups-were articulated spatially into towns and districts. The relationship among these units is far from clear because of the use of the Choctaw term iksa to refer to both the moieties and smaller entities presumed to be lineages or submoiety aggregations of lineages. Early descriptions of the Choctaw show a confusion of names of geographic divisions, moieties, iksas, and local groups. The disruption of Choctaw social organization during the Removal period made it difficult to clarify many points of disagreement. (Galloway et Kidwell 2004, p. 504)

Si O'Brien a raison de rejeter l'idée, ethnologiquement peu informée et pourtant fréquemment avancée ${ }^{32}$, que « each moiety had specific responsibilities, such as one group supplying war leaders and the other providing civil chiefs » (O'Brien 2002, p. 15, 36), les arguments en faveur d'une lecture sociologique du phénomène de division ne sauraient s'y réduire. En l'occurence, ce serait se détourner des propositions de Swanton et de Mary Haas sur la complexité du système chez les Creek (Haas 1940), qui articule au moins deux systèmes duels différents : le premier distribue les groupes de filiation matrilinéaires (imaleikida ou clan) à l'intérieur de chacune des unités géographiques et cérémonielles

commise en quittant les interets des françois » (AN F3, 24, fol. 448v, Lettre de Beauchamp du 24 août 1748).

31. Conception largement influencée semble-t-il par l'ouvrage édité par Maybury-Lewis, dont la présentation du débat et les vues théoriques sur le dualisme en anthropologie ont été jugées durement par Anne-Christine Taylor dans sa recension. Retenons en particulier l'absence de discussion des travaux de Louis Dumont et de la notion d'englobement hiérarchique (Taylor 1993; Dumont 1978).

32. Pour une illustration des effets historiographiques de la transposition aux Chickasaw de cette recette caricaturale (Ethridge 2010, p. 226 et suiv.) et sa critique (Greene 2005). 
(talwa, ou ville), l'autre s'applique à chacun des talwa pris comme entité, dans un système régional aux contours flottants. Haas, dans un souci de clarification, rarement imité, a proposé de désigner uniquement le premier par le terme de "moiety », et de nommer le second "semi-division » - ou " fire clan », pour reprendre l'expression qu'emploient Ellis Childers et Zacharia Cook (Swanton 1928, p. 554, 559), respectivement chefs des talwa de Chiaha et Tukabachee au début du $\mathrm{Xx}^{\mathrm{e}}$ siècle.

La possibilité que nous ayons affaire dans les sources du XVIII ${ }^{e}$ siècle sur les Choctaw à une forme de ce dernier système plutôt qu'au système des moitiés - moins facile à discerner pour des observateurs extérieurs - n'est jamais véritablement discutée ${ }^{33}$. Le modèle des semi-divisions des Creek, illustration d'une forme d'organisation dualiste à l'échelle d'unités territoriales discrètes et l'inscription de cette opposition dans un cérémonialisme aux accents cosmologiques ${ }^{34}$, incite à reprendre la question du dualisme des Choctaw dans la perspective de la dimension politico-rituelle des organisations territoriales. Sans prétendre qu'il suffirait de transposer tel quel le système des semi-divisions des Creek - sur lequel nos vues sont également lacunaires -, pour résoudre instantanément toutes les difficultés posées, il s'agit plutôt de souligner que des sociétés amérindiennes voisines en Amérique du Nord, offrent une grande variété de formes de dualisme qui sont autant d'occasions d'enrichir notre topique anthropologique.

L'organisation des Pawnee des Plaines centrales, par exemple, pourrait offrir un point de comparaison stimulant, car évocateur par bien des aspects de la situation des Choctaw : historiquement structurés en au moins quatre ensembles territoriaux séparés et autonomes ( Skiri-Pawnee, intégrait à travers une organisation essentiellement religieuse ("bundles scheme »), centrée sur des paquets rituels ("bundles ») hiérarchisés, une quinzaine de villages, possédant chacun une forte identité, politique et rituelle, et un système de moitiés en l'absence de segmentation lignagère (Parks 2001).

\section{Conclusion}

Nous admettons cependant qu'améliorer la compréhension du fonctionnement politico-rituel des communautés Choctaw, Chickasaw ou Creek au $\mathrm{XVIII}^{\mathrm{e}}$ siècle, dans une perspective proprement ethnologique est un travail qui

33. C'est cependant le mérite de l'article de discussion que Galloway consacre au livre d'O'Brien d'aborder de façon plus ethnologique cet aspect de la question, y ajoutant également l'idée que certaines oppositions pourraient renvoyer à des classes d'âges (Galloway 2006, p. 366).

34. Ces oppositions se manifestent principalement à l'occasion de rencontres de lacrosse, qui font immédiatement suite à la cérémonie majeure, dite poskita, du feu nouveau. 
reste largement à entreprendre, dont on n'a fait ici qu'ébaucher une orientation possible. Pour aller plus loin, il nous semble que cela devra passer par une évaluation critique de la façon dont l'ethnologie du Sud-Est, influencée par le fonctionnalo-structuralisme d'Alfred Radcliffe-Brown, a eu tendance à réduire le dualisme de ces sociétés à une organisation rigide des formes sociales, un « ordre linéaire univoque », au lieu de chercher à le penser comme idéologie et schème pratique de classement, d'arrangement, de relations assymétriques opérant à différentes échelles (Dumont 1966, p. 111). À travers l'examen des divisions géographiques des Choctaw, nous avons voulu suggérer la possibilité de saisir une forme de continuité politico-rituelle dans ces sociétés, en dépit des modèles historiographiques qui se sont jusqu'ici surtout attachés à mettre l'accent sur l' « effondrement » des modèles sociaux pré-colombiens, entre le $\mathrm{XVI}^{\mathrm{e}}$ siècle et le XVIII ${ }^{\mathrm{e}}$ siècle (Ethridge et Shuck-Hall 2009). Un aspect critique de ce débat nous semble être l'insuffisante appréhension de la dimension rituelle des modèles politiques proprement amérindiens, via notamment la question de la hiérarchie, et sa compatibilité avec différentes manifestations du dualisme (Knight 1990). *

* Manuscrit reçu en décembre 2017, accepté pour publication en novembre 2018.

\section{Références citées}

AN = Archives nationales, séries C13A, F3 et MAR/2JJ.

BALVAY Arnaud

2008 La révolte des Natchez, Le Félin-Kiron (Les marches du temps), Paris.

BLOCH Marc

1997 Apologie pour l'histoire ou Métier d'historien, A. Colin, Paris.

BOCCARA Guillaume

2005 «Mundos nuevos en las fronteras del Nuevo Mundo », Nuevo Mundo Mundos Nuevos. Nouveaux mondes mondes nouveaux [en ligne], 8 février 2005, https://

Boyd Mark F. journals.openedition.org/nuevomundo/426 (consulté le 11/06/19).

1937 «The expedition of Marcos Delgado from Apalache to the Upper Creek Country in 1686 », The Florida Historical Quarterly, 16 (1), p. 2-32.

Chaudhuri Jean et Joyotpaul Chaudhuri

2001 A sacred path the way of the Muscogee Creeks, UCLA American Indian Studies Center, Los Angeles [2 éd.].

Cumming William Patterson

1998 The Southeast in early maps, University of North Carolina Press, Chapel Hill [ $3^{\text {e }}$ éd.].

DEMARIGNY

1743 «Carte particulière d'une partie de la Louisianne ou les fleuve et rivierres [i.e. rivières] onts etés relevé a l'estime $\&$ les routtes [i.e. routes] par terre 
relevé \& mesurées aux pas, par les Srs. Broutin, de Vergés, ingénieurs \& Saucier dessinateur », Library of Congress, Geography and Map Division, G4010 1743.D4, Washington (DC), https://www.loc.gov/resource/g4010.ct0 $00681 /$ ? $\mathrm{r}=-0.799,-0.065,2.598,1.294,0$ (consulté le 13/06/2019).

Demoule Jean-Paul

2014 Mais où sont passés les Indo-Européens? Le mythe d'origine de l'Occident, Édition du Seuil (La Librairie du XXI ${ }^{\mathrm{e}}$ siècle), Paris.

DésveAux Emmanuel

2001 Quadratura americana. Essai d'anthropologie lévi-straussienne, Georg (Ethnos), Genève.

Dumont Louis

1966 Homo hierarchicus. Essai sur le système des castes, Gallimard, Paris.

1978 «La communauté anthropologique et l'idéologie », L'Homme, 18 (3), p. 83-110.

ETHRIDGE Robbie F.

2010 From Chicaza to Chickasaw the European invasion and the transformation of the Mississippian world, 1540-1715, University of North Carolina Press, Chapel Hill.

Ethridge Robbie F. et Sheri M. SHuck-Hall

2009 Mapping the Mississippian shatter zone. The colonial Indian slave trade and regional instability in the American South, University of Nebraska Press, Lincoln.

Galloway Patricia

1989 " "The Chief Who is your Father": Choctaw and French view of the diplomatic relation », in Peter H. Wood, Gregory A. Waselkov et Thomas Hatley (dir.), Powhatan's mantle Indians in the colonial Southeast, University of Nebraska Press (Indians of the Southeast), Lincoln, p. 254-278.

1994 " "So many little republics": British negotiations with the Choctaw Confederacy, $1765 »$ Ethnohistory, 41 (4), p. 513-537.

1995 Choctaw genesis, 1500-1700, University of Nebraska Press (Indians of the Southeast), Lincoln.

2006 Practicing ethnohistory. Mining archives, hearing testimony, constructing narrative, University of Nebraska Press, Lincoln.

Galloway Patricia et Clara S. Kidwell

2004 "Choctaw in the East », in William C. Sturtevant, et Raymond Fogelson (dir.), Handbook of North American Indians, Vol. 14. Southeast, Government Printing Office, Washington (DC), p. 499-519.

GATSChET Albert S.

1884 A migration legend of the Creek Indians, with a linguistic, historic and ethnographic introduction, D. G. Brinton (Brinton's Library of Aboriginal American Litterature, 4), Philadelphia.

Ginzburg Carlo

1999 «Alien Voices », in History, rhetoric, and proof. The Menachem Stern lectures in history, University Press of New England, Hanover, p. 77-91.

2010 Le fil et les traces: vrai faux fictif, Verdier, Lagrasse. 
GinzBurg Carlo

2013 « Nos mots et les leurs. Une réflexion sur le métier de l'historien, aujourd'hui », Fabula, la recherche en littérature [en ligne], http:/www.fabula.org/atelier. php?Ginzburg_Nos_mots_et_les_leurs\#_ftnref6 (consulté le 17/06/2019).

2015 "La longue durée, à la loupe », Conférences des 4 au 26 mai 2015 [en ligne], conférences des 4, 11, 18 et 26 mai 2015 au Collège de France, https://www. college-de-france.fr/site/roger-chartier/guestlecturer-2015-05-04-17h00.htm (consulté le 17/06/2019).

Giudicelli Christophe

2010 "Hétéronomie et classifications coloniales. La construction des "nations" indiennes aux confins de l'Amérique espagnole (XVI-XVII siècle) », Nuevo Mundo Mundos Nuevos. Nouveaux mondes mondes nouveaux [en ligne], 29 mars 2010, https://journals.openedition.org/nuevomundo/59411 (consulté le $17 / 06 / 19)$.

GREENE Richard

2005 «The Red and the White», Chickasaw Times, October 2005, p. 29, 32.

HAAs Mary R.

1940 «Creek Inter-Town Relations », American Anthropologist, 42 (3), p. 479-489.

HANN John H.

1988 Apalachee. The Land between the rivers, University Presses of Florida (Ripley P. Bullen Monographs in Anthropology and History), Gainesville.

HAVARD Gilles

2009 «"Les forcer à devenir Cytoyens". État, sauvages et citoyenneté en NouvelleFrance (XVII ${ }^{\mathrm{e}}-\mathrm{XVIII}$ e siècle) », Annales. Histoire, Sciences Sociales, 64 (5), p. $985-1018$.

2016 Histoire des coureurs de bois Amérique du Nord, 1600-1840, Les Indes savantes (Rivages des Xantons), Paris.

HewitT John N. B.

1939 Notes on the Creek Indians, Bureau of American Ethnology (Bulletin - Smithsonian Institution, 73), Washington (DC).

Hudson Charles

2005 The Juan Pardo expeditions. Exploration of the Carolinas and Tennessee, 1566-1568, University of Alabama Press, Tuscaloosa.

Hudson Charles M. et Carmen Chaves Tesser

1994 The forgotten centuries. Indians and Europeans in the American South, 15211704, University of Georgia Press, Athens.

\section{KNIGHT Vernon}

1990 «Social organization and the evolution of hierarchy in Southeastern Chiefdoms », Journal of Anthropological Research, 46 (1), p. 1-23.

Kowalewski Stephen A.

2006 «Coalescent societies », in Thomas J. Pluckhahn et Robbie Ethridge (dir.), Light on the path. The anthropology and history of the Southeastern Indians, University of Alabama Press, Tuscaloosa, p. 94-122.

NAIRNE Thomas

1988 Nairne's Muskhogean journals. The 1708 expedition to the Mississippi River, University Press of Mississippi, Jackson. 
O’BriEn Greg

2002 Choctaws in a revolutionary age, 1750-1830, University of Nebraska Press (Indians of the Southeast), Lincoln/London.

PARKs Douglas R.

2001 «Pawnee », in Raymond J. Demallie (dir.), Handbook of North American Indians, Vol. 13. Plains, Government Printing Office, Washington (DC), p. 515-547.

Penicaut André Joseph

1721 Relation, ou annale véritable..., [Manuscrit], [en ligne], BNF, Français 14613, http://gallica.bnf.fr/ark:/12148/bpt6k109469c (consulté le 17/06/19).

Piker Joshua A.

2004 Okfuskee, a Creek Indian town in colonial America, Harvard University Press, Cambridge.

Rowland Dunbar

1911 Mississippi provincial archives, English dominion, Vol. I. 1763-1766, Press of Brandon Printing Company, Nashville.

SARDAN Jean-Pierre Olivier de

1998 «Émique », L'Homme, 38 (147), p. 151-166.

SwANTON John R.

1918 «An early account of the Choctaw Indians », in Memoirs of the American Anthropological Association, American Anthropological Association, 5 (2), Lancaster, p. 51-72.

1928 Religious beliefs and medical practices of the Creek Indians, Governement Printing Office (Annual report of the Bureau of American Ethnology to the Secretary of the Smithsonian Institution, 42), Washington (DC), p. 473-672.

1929 Myths and tales of the southeastern Indians, Bureau of American Ethnology (Bulletin. Smithsonian Institution, 88), Washington (DC).

1931 Source material for the social and ceremonial life of the Choctaw Indians, Bureau of American Ethnology (Bulletin. Smithsonian Institution, 103), Washington (DC).

1932 «Choctaw Moieties », American Anthropologist, 34 (2), p. 357-357.

1946 The Indians of the Southeastern United States, Bureau of American Ethnology (Bulletin. Smithsonian Institution, 137), Washington (DC).

TAYlor Anne Christine

1993 «D. Maybury-Lewis \& U. Almagor (eds.), The Attraction of Opposites. Thought and Society in the Dualistic Mode » [compte rendu], L'Homme, 33 (126), p. 539-540.

VEYne Paul

1971 Comment on écrit l'histoire, Éditions du Seuil, Paris.

ViLLIERS Marc de

1907 «Rapport du Chevalier de Kerlérec. Gouverneur de la Louisiane Française. Sur les peuplades des vallées du Mississippi et du Missouri », in Congrès International des Américanistes. XVe session. Tenue à Québec en 1906, Dussault \& Proulx, Québec, p. 61-86.

1922a «Documents concernant l'histoire des Indiens de la région orientale de la Louisiane », Journal de la Société des américanistes, 14 (1), p. 127-140. 
Benjamin BALLOY

VILLIERS Marc de

1922b « Notes sur les Chactas d'après les journaux de voyage de Régis du Roullet (1729-1732) », Journal de la Société des américanistes, 14 (1), p. 223-250.

WASELKOv Gregory A.

1989 «Indian maps of the Colonial Southeast», in Peter H. Wood, Gregory A. Waselkov et Thomas Hatley (dir.), Powhatan's mantle Indians in the colonial Southeast, University of Nebraska press (Indians of the Southeast), Lincoln.

WENHOLD Lucy

1936 A 17th Letter of Gabriel Diaz Vara Calderon, Bishop of Cuba, describing the Indians and Indian Missions of Florida, Smithsonian Institution (Smithsonian miscellaneous collections, 95 [16]), Washington (DC).

White Richard

2009 Le Middle ground. Indiens, empires et républiques dans la région des Grands Lacs, 1650-1815, Anacharsis, Toulouse. 